\title{
Numerical algorithm for solving second order nonlinear fuzzy initial value problems
}

\author{
A. F. Jameel' ${ }^{1}$, N.R. Anakira ${ }^{2}$, A. H. Shather ${ }^{3}$, Azizan Saaban ${ }^{4}$, A. K. Alomari ${ }^{5}$ \\ ${ }^{1,4}$ School of Quantitative Sciences, Universiti Utara Malaysia (UUM), Malaysia \\ ${ }^{2}$ Department of Mathematics Faculty of Science and Technology Irbid National University, Jordan \\ ${ }^{3}$ Department of Computer Engineering Techniques, Al Kitab University College, Iraq \\ ${ }^{5}$ Department of Mathematics, Faculty of Science, Yarmouk University, Jordan
}

\begin{tabular}{l}
\hline \hline Article Info \\
\hline Article history: \\
Received Aug 23, 2019 \\
Revised May 25, 2020 \\
Accepted Jun 5, 2020 \\
\hline Keywords: \\
FIVP \\
Fuzzy differential equations \\
Fuzzy set theory \\
RK56 \\
Second-order nonlinear \\
Six stages runge kutta
\end{tabular}

\begin{abstract}
The purpose of this analysis would be to provide a computational technique for the numerical solution of second-order nonlinear fuzzy initial value (FIVPs). The idea is based on the reformulation of the fifth order Runge Kutta with six stages (RK56) from crisp domain to the fuzzy domain by using the definitions and properties of fuzzy set theory to be suitable to solve second order nonlinear FIVP numerically. It is shown that the second order nonlinear FIVP can be solved by RK56 by reducing the original nonlinear equation intoa system of couple first order nonlinear FIVP. The findings indicate that the technique is very efficient and simple to implement and satisfy the Fuzzy solution properties. The method's potential is demonstrated by solving nonlinear second-order FIVP.
\end{abstract}

Copyright $@ 2020$ Institute of Advanced Engineering and Science. All rights reserved.

\section{Corresponding Author:}

A. F. Jameel,

School of Quantitative Sciences,

Universiti Utara Malaysia (UUM),

Kedah, Sintok, 06010 Malaysia.

Email: homotopy33@gmail.com

\section{INTRODUCTION}

As a conceptual model, several complex real-life problems can be articulated. Such models' equations may either be constructed as an ordinary or partial differential equation $[1,2]$. These equations can be solved via several method numerical or approximated method [3-6]. The FDEs are helpful in modelling a complex structure when knowledge is insufficient on its behavior. The FIVPs arises as such models have not been developed and are unpredictable in nature. These types of models can be defined by mathematical dynamic systems models under vagueness of FDEs. Real words, phenomena such as population physics and medicine take into account of fuzzy problems [5-9]. Ordinary differential. The efficacy of the methods proposed can be tested on linear FIVPs as simplest consideration. The dynamics of the Range Kutta methods depend on increasing number calculations in the methods function per each step that we can gain the method's ability to solve such problems [10].

The explicit fourth order Runge-Kutta methods have been widely used in the solution for first and high order FIVPs $[11,12]$. Various types of RungeKutte methods have been used in the last ducat to solve linear second order FIVPs, including the second order RungeKutta method [10], the third order Runge Kutta methods [13], and the fifth order Runge Kutta method of five stages in [14]. The focus is to develop and evaluate RK56's use to provide the non-linear second order FIVP numerical solution. This is the first attempt, to the best of our knowledge, to solve the nonlinear form of second order FIVP using RK56. The present article shall be arranged accordingly. We start with certain standard concepts for fuzzy numbers in section 2 . 
The second order FIVP fuzzy analysis is described in section 3. We analyze and develop RK56 for the solution of the second order of FIVP in section 4. For the nonlinear second order FIVP solution, section 5 uses RK56 for a test example and display results in the form of table and figures. Finally, in section 6, we give the conclusion of this study.

\section{PRELIMINARIES}

Some definitions and most basic notes are standard and popular (see [15-19] for details), in this section, which will be used in this paper, are introduced

Definition 2.1([20]): "The triangular fuzzy are linked to degrees of membership which state how true it is to say if something belongs or not to a determined set. As shown if Figure 1 this number is defined by three parameters $\alpha<\beta<\gamma$ where the graph of $\mu(x)$ is a triangle with the base on the interval $[\alpha, \beta]$ and vertex at $\mathrm{x}=\beta$ and its membership function has the following form:

$$
\mu(x ; \alpha, \beta, \gamma)=\left\{\begin{aligned}
0, & \text { if } x<\alpha \\
\frac{x-\alpha}{\beta-\alpha}, & \text { if } \alpha \leq x \leq \beta \\
\frac{\gamma-x}{\gamma-\beta}, & \text { if } \beta \leq x \leq y \\
0, & \text { if } x>\gamma
\end{aligned}\right.
$$

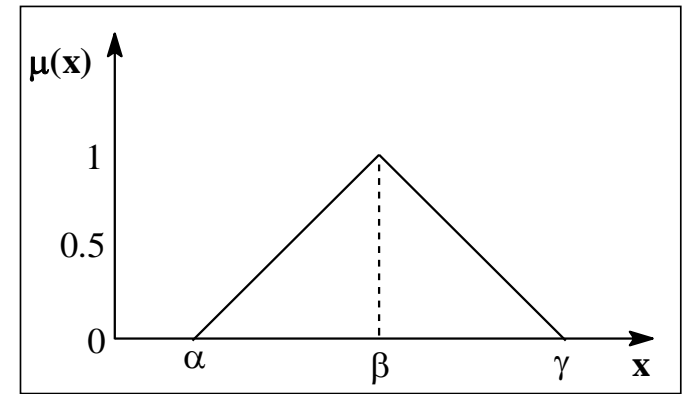

Figure 1. Triangular fuzzy number

and its r-level is: $[\mu]_{\mathrm{r}}=[\alpha+\mathrm{r}(\beta-\alpha), \gamma-\mathrm{r}(\gamma-\beta)], \mathrm{r} \in[0,1]$.

Definition 2.2([4]): "A fuzzy number is a function $\mathbb{R}: \mathrm{R} \rightarrow[0,1]$ satisfying the following properties:

1. $\mu(\mathrm{x})$ is normal, i.e $\exists \mathrm{t}_{0} \in \mathbb{R}$ with $\mu\left(\mathrm{t}_{0}\right)=1$,

2. $\mu(\mathrm{x})$ Is convex fuzzy set, i. e. $\mu(\lambda \mathrm{x}+(1-\lambda) \mathrm{t}) \geq \min \{\mu(\mathrm{x}), \mu(\mathrm{t})\} \forall \mathrm{x}, \mathrm{t} \in \mathbb{R}, \lambda[0,1]$,

3. $\mu$ upper semi-continuous on $\mathbb{R}$,

4. $\{t \in \mathbb{R}: \mu(x)>0\}$ is compact.

$\widetilde{\mathrm{E}}$ is called the space of fuzzy numbers and $\mathbb{R}$ is a proper subset of $\widetilde{\mathrm{E}}$.

Define the r-level set $x \in \mathbb{R},[\mu]_{\mathrm{r}}=\{\mathrm{x}: \mu(\mathrm{x}) \geq \mathrm{r}\}, 0 \leq \mathrm{r} \leq 1$ where $[\mu]_{0}=\{\mathrm{x}: \mu(\mathrm{x})>0\}$ compact, which is a closed bounded interval and denoted by is $[\mu]_{r}=[\underline{\mu}(\mathrm{x}), \bar{\mu}(\mathrm{x})]$. In the single parametric form [2], a fuzzy number is represented by an ordered pair of functions $[\underline{\mu}(\mathrm{x}), \bar{\mu}(\mathrm{x})], \mathrm{r} \in[0,1]$ which satisfies:

1. $\mu(\mathrm{x})$ is a bounded left continuous non-decreasing function over $[0,1]$.

2. $\bar{\mu}(\mathrm{x})$ is a bounded left continuous non-increasing function over $[0,1]$.

3. $\underline{\mu}(\mathrm{x}) \leq \bar{\mu}(\mathrm{x}), \mathrm{r} \in[0,1]$. A crisp number $\mathrm{r}$ is simply represented by $\underline{\mu}(\mathrm{r})=\bar{\mu}(\mathrm{r})=\mathrm{r}, \mathrm{r} \in[0,1] . "$

Definition 2.2([4]) "If $\widetilde{\mathrm{E}}$ be the set of all fuzzy numbers, we say that $\mathrm{f}(\mathrm{x})$ is a fuzzy function if $\mathrm{f}: \mathbb{R} \rightarrow \widetilde{\mathrm{E}}$ "

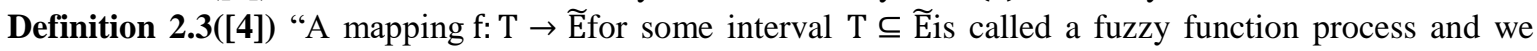
denote r-level set by:

$$
[\tilde{\mathrm{f}}(\mathrm{x})]_{\mathrm{r}}=[\underline{\mathrm{f}}(\mathrm{x} ; \mathrm{r}), \overline{\mathrm{f}}(\mathrm{x} ; \mathrm{r})], \mathrm{x} \in \mathrm{K}, \mathrm{r} \in[0,1]
$$


The r-level sets of a fuzzy number are much more effective as representation forms of fuzzy set than the above. Fuzzy sets can be defined by the families of their r-level sets based on the resolution identity theorem."

Definition 2.5([18]): Each function $\mathrm{f}: \mathrm{X} \rightarrow \mathrm{Y}$ induces another function $\tilde{\mathrm{f}}: \mathrm{F}(\mathrm{X}) \rightarrow \mathrm{F}(\mathrm{Y})$ defined for each fuzzy interval U in X by:

$$
\tilde{\mathrm{f}}(\mathrm{U})(\mathrm{y})= \begin{cases}\operatorname{Sup}_{\mathrm{x} \in \mathrm{f}^{-1}(\mathrm{y})} \mathrm{U}(\mathrm{x}) & , \text { if } \mathrm{y} \in \operatorname{range}(\mathrm{f}) \\ 0 & \text { if } \mathrm{y} \notin \operatorname{range}(\mathrm{f})\end{cases}
$$

This is called the Zadeh extension principle.

Definition 2.6 ([4]): “Consider $\tilde{x}, \tilde{y} \in \widetilde{E}$. If there exists $\tilde{z} \in \widetilde{E}$ such that $\tilde{x}=\tilde{y}+\tilde{z}$, then $\tilde{z}$ is called the H-difference (Hukuhara difference) of $\mathrm{x}$ and $\mathrm{y}$ and is denoted by $\tilde{z}=\tilde{\mathrm{x}} \ominus \tilde{\mathrm{y}}$."

Definition 2.7([4]) "If $\tilde{\mathrm{f}} \mathrm{I} \rightarrow \widetilde{\mathrm{E}}$ and $\mathrm{y}_{0} \in \mathrm{I}$, where $\mathrm{I} \in\left[\mathrm{x}_{0}, \mathrm{~K}\right]$. We say that $\tilde{\mathrm{f}}$ Hukuhara differentiable at $\mathrm{y}_{0}$,


$\tilde{\mathrm{f}}\left(\mathrm{y}_{0} ; \mathrm{r}\right), \tilde{\mathrm{f}}\left(\mathrm{y}_{0} ; \mathrm{r}\right) \ominus \tilde{\mathrm{f}}\left(\mathrm{y}_{0}-\mathrm{h} ; \mathrm{r}\right)$ and the limits are taken in the metric $(\widetilde{\mathrm{E}}, \mathrm{D})$ :

$$
\lim _{\mathrm{h} \rightarrow 0+} \frac{\tilde{\mathrm{f}}\left(\mathrm{y}_{0}+\mathrm{h} ; \mathrm{r}\right) \Theta \tilde{\mathrm{f}}\left(\mathrm{y}_{0} ; \mathrm{r}\right)}{\mathrm{h}}=\lim _{\mathrm{h} \rightarrow 0+} \frac{\tilde{\mathrm{f}}\left(\mathrm{y}_{0} ; \mathrm{r}\right) \Theta \tilde{\mathrm{f}}\left(\mathrm{y}_{0}-\mathrm{h} ; \mathrm{r}\right)}{\mathrm{h}}
$$

The fuzzy set $\left[\widetilde{\mathrm{f}^{\prime}}\left(\mathrm{y}_{0}\right)\right]_{\mathrm{r}}$ is called the Hukuhara derivative of $\left[\tilde{\mathrm{f}}^{\prime}\right]_{\mathrm{r}}$ at $\mathrm{y}_{0}$.

These limits are taken in the space $(\widetilde{E}, D)$ if $x_{0}$ or $K$, then we consider the corresponding one-side derivation. Recall that $\tilde{\mathrm{x}} \ominus \tilde{\mathrm{y}}=\tilde{\mathrm{z}} \in \widetilde{\mathrm{E}}$ are defined on r-level set, where $[\tilde{\mathrm{x}}]_{\mathrm{r}} \ominus[\tilde{\mathrm{y}}]_{\mathrm{r}}=[\tilde{\mathrm{z}}]_{\mathrm{r}}, \forall \mathrm{r} \in[0,1]$ By consideration of definition of the metric D all the r-level set $[\tilde{f}(0)]_{r}$ are Hukuhara differentiable at $y_{0}$,

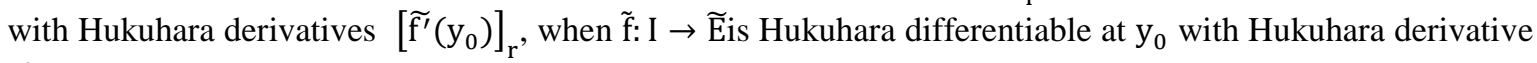
$\left[\widetilde{\mathrm{f}^{\prime}}\left(\mathrm{y}_{0}\right)\right]_{\mathrm{r}}$ it' lead to that $\tilde{\mathrm{f}}$ is Hukuhara differentiable for all $\mathrm{r} \in[0,1]$ which satisfies the above limits i.e. if $\mathrm{f}$ is differentiable at $\mathrm{x}_{0} \in\left[\mathrm{x}_{0}+\alpha, \mathrm{K}\right]$ then all its r-levels $\left[\widetilde{\mathrm{f}^{\prime}}(\mathrm{x})\right]_{\mathrm{r}}$ are Hukuhara differentiable at $\mathrm{x}_{0}$."

Definition 2.8([4]) "Define the mapping $\widetilde{\mathrm{f}^{\prime}}: \mathrm{I} \rightarrow \widetilde{\mathrm{E}}$ and $\mathrm{y}_{0} \in \mathrm{I}$, where $\mathrm{I} \in\left[\mathrm{x}_{0}, \mathrm{~K}\right]$. We say that $\widetilde{\mathrm{f}^{\prime}}$ Hukuhara differentiable $\mathrm{x} \in \widetilde{\mathrm{E}}$, if there exists an element $\left[\tilde{\mathrm{f}}^{(\mathrm{n})}\right]_{\mathrm{r}} \in \widetilde{\mathrm{E}}$ such that for allh $>0$ sufficiently small (near to $0)$, exists $\tilde{\mathrm{f}}^{(\mathrm{n}-1)}\left(\mathrm{y}_{0}+\mathrm{h} ; \mathrm{r}\right) \ominus \tilde{\mathrm{f}}^{(\mathrm{n}-1)}\left(\mathrm{y}_{0} ; \mathrm{r}\right), \tilde{\mathrm{f}}^{(\mathrm{n}-1)}\left(\mathrm{y}_{0} ; \mathrm{r}\right) \ominus \tilde{\mathrm{f}}^{(\mathrm{n}-1)}\left(\mathrm{y}_{0}-\mathrm{h} ; \mathrm{r}\right)$ and the limits are taken in the metric $(\widetilde{E}, D)$

$$
\lim _{\mathrm{h} \rightarrow 0+} \frac{\tilde{\mathrm{f}}^{(\mathrm{n}-1)}\left(\mathrm{y}_{0}+\mathrm{h} ; \mathrm{r}\right) \Theta \tilde{\mathrm{f}}^{(\mathrm{n}-1)}\left(\mathrm{y}_{0} ; \mathrm{r}\right)}{\mathrm{h}}=\lim _{\mathrm{h} \rightarrow 0+} \frac{\tilde{\mathrm{f}}^{(\mathrm{n}-1)}\left(\mathrm{y}_{0} ; \mathrm{r}\right) \Theta \tilde{\mathrm{f}}^{(\mathrm{n}-1)}\left(\mathrm{y}_{0}-\mathrm{h} ; \mathrm{r}\right)}{\mathrm{h}}
$$

exists and equal to $\tilde{\mathrm{f}}^{(\mathrm{n})}$ and for $\mathrm{n}=2$ we have second order Hukuharaderivative."

Theorem 2.2([4]) “ Let $\tilde{\mathrm{f}}:\left[\mathrm{x}_{0}+\alpha, \mathrm{K}\right] \rightarrow \widetilde{\mathrm{E}}$ be Hukuhara differentiable and denote

$$
\left[\widetilde{\mathrm{f}^{\prime}}(\mathrm{x})\right]_{\mathrm{r}}=\left[\mathrm{f}^{\prime}(\mathrm{x}), \overline{\mathrm{f}}^{\prime}(\mathrm{x})\right]_{\mathrm{r}}=\left[\underline{\mathrm{f}}^{\prime}(\mathrm{x} ; \mathrm{r}), \overline{\mathrm{f}}^{\prime}(\mathrm{x} ; \mathrm{r})\right]
$$

Then the boundary functionsf $\underline{f}^{\prime}(\mathrm{x} ; \mathrm{r}), \overline{\mathrm{f}}^{\prime}(\mathrm{x} ; \mathrm{r})$ are differentiable we can write for second order fuzzy derivative

$$
\left[\tilde{\mathrm{f}}^{\prime \prime}(\mathrm{x})\right]_{\mathrm{r}}=\left[\left(\underline{\mathrm{f}}^{\prime \prime}(\mathrm{x} ; \mathrm{r})\right)^{\prime},\left(\overline{\mathrm{f}}^{\prime \prime}(\mathrm{x} ; \mathrm{r})\right)^{\prime}\right], \forall \mathrm{r} \in[0,1]
$$

\section{ANALYSIS OF SECOND ORDER FIVP}

Consider the second order nonlinear FIVP of the following form:

$$
\tilde{y}^{\prime \prime}(x)=\tilde{\mathcal{N}}\left(\tilde{y}(x), \tilde{y}^{\prime}(x)\right)+\tilde{n}(x), \quad x \in\left[x_{0}, K\right]
$$

Subject to the fuzzy initial conditions 


$$
\tilde{y}\left(x_{0}\right)=\tilde{\alpha}_{0}, \tilde{y}^{\prime}\left(x_{0}\right)=\tilde{\beta}_{0}
$$

where the defuzzification details can be found in [4]:

" $\tilde{y}(x)$ : is a fuzzy function in terms of $x$ such that $[\tilde{y}(x)]_{r}=\left[y(x)^{(r)}, \bar{y}(x)^{(r)}\right]$,

$\tilde{y}^{\prime}(x)$ : is the first order fuzzy Hukuhara-derivative such that $\left[\tilde{y}^{\prime}(x)\right]_{r}=\left[\underline{y}^{\prime}(x)^{(r)}, \bar{y}^{\prime}(x)^{(r)}\right]$,

$\tilde{y}^{\prime \prime}(x)$ : is the second order fuzzy Hukuhara-derivative such that $\left[\tilde{y}^{\prime \prime}(x)\right]_{r}=\left[\underline{y}^{\prime \prime}(x)^{(r)}, \bar{y}^{\prime \prime}(x)^{(r)}\right]$,

$\widetilde{\mathcal{N}}$ : is fuzzy nonlinear function in terms of $x$, the fuzzy variable $\tilde{y}$ and the fuzzy derivative $\tilde{y}^{\prime}$,"

From the above analysis we define the following relations:

$$
\begin{aligned}
& {\left[\tilde{\mathcal{N}}\left(\tilde{y}(x), \tilde{y}^{\prime}(x)\right)\right]_{r}=\left[\underline{\mathcal{N}}\left(x, \underline{y}, \underline{y}^{\prime}\right)^{(r)}, \overline{\mathcal{N}}\left(x, \bar{y}, \bar{y}^{\prime}\right)^{(r)}\right] \text {, such that }} \\
& \tilde{n}(x)=\tilde{p} t(x)+\tilde{q}
\end{aligned}
$$

where the nonhomogeneous term is $[\tilde{n}(x)]_{r}=\left[\underline{n}(x)^{(r)}, \bar{n}(x)^{(r)}\right]$ such that $\tilde{p} t(x)+\tilde{q}$. The fuzzy coefficients in (2) are triangular fuzzy numbers denoted by $[\tilde{p}]_{r}=[\underline{p}, \bar{p}]_{r},[\tilde{q}]_{r}=[\underline{q}, \bar{q}]_{r}$ for all the fuzzy level set $r \in[0,1]$.

$\tilde{y}_{0}:$ is the first fuzzy initial condition triangular numbers denoted by $\left[\tilde{y}\left(x_{0}\right)\right]_{r}=\left[\underline{y}\left(x_{0}\right)^{(r)}, \bar{y}\left(x_{0}\right)^{(r)}\right]$, such that $\left[\tilde{\alpha}_{0}\right]_{r}=\left[\underline{\alpha}_{0}, \bar{\alpha}_{0}\right]_{r}$, finally:

$\tilde{y}^{\prime}:$ is the second fuzzy initial condition triangular numbers denoted by $\left[\tilde{y}^{\prime}\left(x_{0}\right)\right]_{r}=\left[\underline{y}^{\prime}\left(x_{0}\right)^{(r)}, \bar{y}^{\prime}\left(x_{0}\right)^{(r)}\right]$. such that $\left[\tilde{\beta}_{0}\right]_{r}=\left[\underline{\beta}_{0}, \bar{\beta}_{0}\right]_{r}$.

Also, we can write

$$
\left\{\begin{array}{l}
\underline{\mathcal{N}}\left(\tilde{y}(x), \tilde{y}^{\prime}(x)\right)^{(r)}=L\left[\underline{y}, \bar{y}, \underline{y}^{\prime}, \bar{y}^{\prime}\right]_{r} \\
\overline{\mathcal{N}}\left(\tilde{y}(x), \tilde{y}^{\prime}(x)\right)^{(r)}=U\left[\underline{y}, \bar{y}, \underline{y}^{\prime}, \bar{y}^{\prime}\right]_{r}
\end{array}\right.
$$

Let

$$
\begin{aligned}
& \tilde{\mathcal{N}}\left(\tilde{y}(x), \tilde{y}^{\prime}(x)\right)+\tilde{n}(t)=\tilde{G}\left(x, \tilde{y}(x), \tilde{y}^{\prime}(x)\right), \\
& \tilde{S}(x)=\tilde{y}(x), \tilde{y}^{\prime}(x),
\end{aligned}
$$

where $[\tilde{S}(x)]_{r}=\left[\underline{S}(x)^{(r)}, \bar{S}(x)^{(r)}\right]$ such that $\tilde{y}^{\prime \prime}(t)=\tilde{G}(x, \tilde{S}(x))$ and from definition 2.5, we can define the following relation:

$$
\left\{\begin{array}{l}
\underline{G}(x, \tilde{S}(x))^{(r)}=\min \{\tilde{G}(x, \tilde{\mu}(r)) \mid \tilde{\mu}(r) \in \tilde{y}(x)\} \\
\bar{G}(x, \tilde{S}(x))^{(r)}=\max \{\tilde{G}(x, \tilde{\mu}(r)) \mid \tilde{\mu}(r) \in \tilde{y}(x)\}
\end{array}\right.
$$

where

$$
\left\{\begin{array}{l}
\underline{G}(x, \tilde{S}(x))^{(r)}=L^{*}[x, \tilde{S}(x)]_{r}=L^{*}\left(x, \tilde{S}(x)^{(r)}\right) \\
\bar{G}(x, \tilde{S}(x))^{(r)}=U^{*}[x, \tilde{S}(x)]_{r}=U^{*}\left(x, \tilde{S}(x)^{(r)}\right)
\end{array}\right.
$$

From (7) and (8) we rewrite (1) as follows

$$
\begin{aligned}
& \left\{\begin{array}{c}
\underline{y}^{\prime \prime}(x)^{(r)}=L^{*}\left(t, \tilde{S}(x)^{(r)}\right) \\
\underline{y}\left(x_{0}\right)^{(r)}=\underline{\alpha}_{0}, \underline{y}^{\prime}\left(x_{0}\right)^{(r)}=\underline{\beta}_{0}
\end{array}\right. \\
& \left\{\begin{array}{c}
\bar{y}^{\prime \prime}(x)^{(r)}=U^{*}\left(t, \tilde{S}(x)^{(r)}\right) \\
\bar{y}\left(x_{0}\right)^{(r)}=\underline{\alpha}_{0}, \bar{y}^{\prime}\left(x_{0}\right)^{(r)}=\bar{\beta}_{0}
\end{array}\right.
\end{aligned}
$$




\section{4. $\quad$ ANALYSIS OF FUZZY RK56}

Here we take into account the six steps Runge Kutta of order five process as a framework for obtaining a numerical solution of second-order FIVP. In [21-23] the structure of this method to solve the crisp initial values problems of was introduced. Also in [24] RK56 to solve first order linear FIVPs with fuzzy intial conditions. In addition, we must defuzzify RKM56 in order to solve nonlinear second order FIVPs. We consider (1) in section 3 and since RK56 is numerical method we need to reduce (1) in to system of first order nonlinear FIVPs for all $\in[0,1]$ :

$$
\left\{\begin{array}{c}
\tilde{y}^{\prime}{ }_{1}(x)^{(r)}=\tilde{G}_{1}\left(t, \tilde{S}(x)^{(r)}\right) \\
\tilde{y}^{\prime}{ }_{2}(x)^{(r)}=\tilde{G}_{2}\left(t, \tilde{S}(x)^{(r)}\right) \\
\tilde{y}_{1}\left(x_{0}\right)^{(r)}=\tilde{\alpha}_{0}(r)=\tilde{a}_{1}, \tilde{y}_{2}\left(x_{0}\right)^{(r)}=\tilde{\beta}_{0}(r)
\end{array}\right.
$$

where $x \in\left[x_{0}, K\right]$, and $\tilde{\alpha}_{0}, \tilde{\beta}_{0}$ are fuzzy numbers for all $r \in[0,1]$ such that

$$
\tilde{y}_{1,0}(r)=\left[\underline{y}_{1,0}(r), \bar{y}_{1,0}(r)\right]=\left[\underline{\alpha}_{0}, \bar{\alpha}_{0}\right]_{r}, \tilde{y}_{2,0}(r)=\left[\underline{y}_{2,0}(r), \bar{y}_{2,0}(r)\right]=\left[\underline{\beta}_{0}, \bar{\beta}_{0}\right]_{r}
$$

Assume $\tilde{y}(x)^{(r)}=\left[\tilde{y}_{1}(x)^{(r)}, \tilde{y}_{2}(x)^{(r)}\right]^{t}$ be the fuzzy solution of system $(11)$ on $\left[x_{0}, K\right]$ then according to section (3) for $i=1,2$ we have:

$$
\begin{aligned}
& \underline{y}_{i}{ }^{\prime}(x)^{(r)}=\min \left\{\tilde{G}_{i}\left(x, \tilde{y}_{i}(x)^{(r)}\right) ; \tilde{y}_{i}(x)^{(r)} \in\left[\underline{y}_{i}(x)^{(r)}, \bar{y}_{i}(x)^{(r)}\right]=\underline{G}_{i}\left(x, \tilde{y}_{i}(x)^{(r)}\right)\right\} \\
& \bar{y}_{i}{ }^{\prime}(x)^{(r)}=\max \left\{\tilde{G}_{i}\left(x, \tilde{y}_{i}(x)^{(r)}\right) ; \tilde{y}_{i}(x)^{(r)} \in\left[\underline{y}_{i}(x)^{(r)}, \bar{y}_{i}(x)^{(r)}\right]=\bar{G}_{i}\left(x, \tilde{y}_{i}(x)^{(r)}\right)\right\}
\end{aligned}
$$

and

$$
\left\{\begin{array}{l}
{\left[\underline{G}_{i}\left(x, \tilde{y}_{i}(x)^{(r)}\right)\right]^{t}=L^{*}\left(x, \underline{y}_{i}(x)^{(r)}, \bar{y}_{i}(x)^{(r)}\right)=L^{*}\left(x, \tilde{y}_{i}(x)^{(r)}\right)} \\
{\left[\bar{G}_{i}\left(x \tilde{y}_{i}(x)^{(r)}\right)\right]^{t}=U^{*}\left(x, \underline{y}_{i}(x)^{(r)}, \bar{y}_{i}(x)^{(r)}\right)=U^{*}\left(x, \tilde{y}_{i}(x)^{(r)}\right)}
\end{array}\right.
$$

From (11-13), system (10) can be written with following assumptions for $i=1,2$

$$
\left\{\begin{array}{c}
\underline{y}^{\prime}(x)^{(r)}=L^{*}\left(x, \tilde{y}_{i}(x)^{(r)}\right) \\
\underline{y}\left(x_{0}\right)^{(r)}=\underline{y}_{0} \in\left[x_{0}, K\right]
\end{array}\right.
$$

where $\underline{y}\left(x_{0}\right)^{(r)}=\left[\underline{y}_{i, 0}(r)\right]^{t}$ and $\underline{y}^{\prime}(x)^{(r)}=\left[\underline{y}_{i}^{\prime}(x)^{(r)}\right]^{t}$ and

$$
\left\{\begin{array}{c}
\bar{y}^{\prime}\left(x_{0}\right)^{(r)}=U^{*}\left(x, \tilde{y}_{i}(x)^{(r)}\right) \\
\bar{y}\left(x_{0}\right)^{(r)}=\bar{y}_{0} \in\left[x_{0}, K\right]
\end{array}\right.
$$

where $\bar{y}\left(x_{0}\right)^{(r)}=\left[\bar{y}_{i, 0}(r)\right]^{t}$ and $\bar{y}^{\prime}\left(x_{0}\right)^{(r)}=\left[\bar{y}_{i}^{\prime}(x)^{(r)}\right]^{t}$.

Now we show that under the assumption for the fuzzy functions $\tilde{G}_{i}$, for $i=1,2$ solution for system (11) for each $r$-level set can obtain a unique fuzzy [11]. Now, for the analysis of fuzzy RK56 with a view to finding a numerical solution of system (11), we first the followings:

$$
\begin{aligned}
& \tilde{y}(x)^{(r)}=\left[\tilde{y}_{1}(x)^{(r)}, \tilde{y}_{2}(x)^{(r)}\right]^{t} \text { such that: } \\
& \tilde{z}_{1}=\tilde{y}_{1}(x)^{(r)}, \tilde{z}_{2}=\tilde{y}_{2}(x)^{(r)} \text { where } \tilde{z}_{i} \in\left[\underline{z}_{i}, \bar{z}_{i}\right] \text { for } i=1,2 . \\
& \underline{V}_{i 1}\left(x ; \underline{y}(x)^{(r)}\right)=\min \left\{\tilde{G}_{i}\left(x, \tilde{z}_{1}, \tilde{z}_{2}\right) ; \tilde{z}_{i} \in\left[\underline{y}_{i}(x)^{(r)}, \bar{y}_{i}(x)^{(r)}\right]\right\} \\
& \bar{V}_{i 1}\left(x ; \bar{y}(x)^{(r)}\right)=\max \left\{\tilde{G}_{i}\left(x, \tilde{z}_{1}, \tilde{z}_{2}\right) ; \tilde{z}_{i} \in\left[\underline{y}_{i}(x)^{(r)}, \bar{y}_{i}(x)^{(r)}\right]\right\} \\
& \underline{V}_{i 2}\left(x ; \underline{y}(x)^{(r)}\right)=\min \left\{\tilde{G}_{i}\left(x+\frac{h}{2}, \tilde{z}_{1}, \tilde{z}_{2}\right) ; \tilde{z}_{i} \in\left[\underline{s}_{i 1}\left(x, \underline{y}(x)^{(r)}, h\right), \bar{s}_{i 1}\left(x, \bar{y}(x)^{(r)}, h\right)\right]\right\} \\
& \bar{V}_{i 2}\left(x ; \bar{y}(x)^{(r)}\right)=\max \left\{\tilde{G}_{i}\left(x+\frac{h}{2}, \tilde{z}_{1}, \tilde{z}_{2}\right) ; \tilde{z}_{i} \in\left[\underline{s}_{i 1}\left(x, \underline{y}(x)^{(r)}, h\right), \bar{s}_{i 1}\left(x, \bar{y}(x)^{(r)}, h\right)\right]\right\} \\
& \underline{V}_{i 3}\left(x ; \underline{y}(x)^{(r)}\right)=\min \left\{\tilde{G}_{i}\left(x+\frac{h}{4}, \tilde{z}_{1}, \tilde{z}_{2}\right) ; \tilde{z}_{i} \in\left[\underline{s}_{i 2}\left(x, \underline{y}(x)^{(r)}, h\right), \bar{s}_{i 2}\left(x, \bar{y}(x)^{(r)}, h\right)\right]\right\}
\end{aligned}
$$




$$
\begin{aligned}
& \bar{V}_{i 3}\left(x ; \bar{y}(x)^{(r)}\right)=\max \left\{\tilde{G}_{i}\left(x+\frac{h}{4}, \tilde{z}_{1}, \tilde{z}_{2}\right) ; \tilde{z}_{i} \in\left[\underline{s}_{i 2}\left(x, \underline{y}(x)^{(r)}, h\right), \bar{s}_{i 2}\left(x, \bar{y}(x)^{(r)}, h\right)\right]\right\} \\
& \underline{V}_{i 4}\left(x ; \underline{y}(x)^{(r)}\right)=\min \left\{\tilde{G}_{i}\left(x+\frac{h}{2}, \tilde{z}_{1}, \tilde{z}_{2}\right) ; \tilde{z}_{i} \in\left[\underline{s}_{i 3}\left(x, \underline{y}(x)^{(r)}, h\right), \bar{s}_{i 3}\left(x, \bar{y}(x)^{(r)}, h\right)\right]\right\} \\
& \bar{V}_{i 4}\left(x ; \bar{y}(x)^{(r)}\right)=\max \left\{\tilde{G}_{i}\left(x+\frac{h}{2}, \tilde{z}_{1}, \tilde{z}_{2}\right) ; \tilde{z}_{i} \in\left[\underline{s}_{i 3}\left(x, \underline{y}(x)^{(r)}, h\right), \bar{s}_{i 3}\left(x, \bar{y}(x)^{(r)}, h\right)\right]\right\} \\
& \underline{V}_{i 5}\left(x ; \underline{y}(x)^{(r)}\right)=\min \left\{\tilde{G}_{i}\left(x+\frac{3 h}{4}, \tilde{z}_{1}, \tilde{z}_{2}\right) ; \tilde{z}_{i} \in\left[\underline{s}_{i 4}\left(x, \underline{y}(x)^{(r)}, h\right), \bar{s}_{i 4}\left(x, \bar{y}(x)^{(r)}, h\right)\right]\right\} \\
& \bar{V}_{i 5}\left(x ; \bar{y}(x)^{(r)}\right)=\max \left\{\tilde{G}_{i}\left(x+\frac{3 h}{4}, \tilde{z}_{1}, \tilde{z}_{2}\right) ; \tilde{z}_{i} \in\left[\underline{s}_{i 4}\left(x, \underline{y}(x)^{(r)}, h\right), \bar{s}_{i 4}\left(x, \bar{y}(x)^{(r)}, h\right)\right]\right\} \\
& \underline{V}_{i 6}\left(x ; \underline{y}(x)^{(r)}\right)=\min \left\{\tilde{G}_{i}\left(x+h, \tilde{z}_{1}, \tilde{z}_{2}\right) ; \tilde{z}_{i} \in\left[\underline{s}_{i 5}\left(x, \underline{y}(x)^{(r)}, h\right), \bar{s}_{i 5}\left(x, \bar{y}(x)^{(r)}, h\right)\right]\right\} \\
& \bar{V}_{i 6}\left(x ; \bar{y}(x)^{(r)}\right)=\max \left\{\tilde{G}_{i}\left(x+h, \tilde{z}_{1}, \tilde{z}_{2}\right) ; \tilde{z}_{i} \in\left[\underline{s}_{i 5}\left(x, \underline{y}(x)^{(r)}, h\right), \bar{s}_{i 5}\left(x, \bar{y}(x)^{(r)}, h\right)\right]\right\}
\end{aligned}
$$

where

$$
\begin{array}{r}
\underline{s}_{i 1}\left(x ; \underline{y}(x)^{(r)}, h\right)=\underline{y}_{i}(x)^{(r)}+\frac{h}{2} \underline{V}_{i 1}(r), \bar{s}_{i 1}\left(x, \bar{y}(x)^{(r)}, h\right)=\bar{y}_{i}(x)^{(r)}+\frac{h}{2} \bar{V}_{i 1}(r) \\
\underline{s}_{i 2}\left(x ; \underline{y}(x)^{(r)}, h\right)=\underline{y}_{i}(x)^{(r)}+\frac{h}{16}\left(3 \underline{V}_{i 1}(r)+\underline{V}_{i 2}(r)\right) \\
\bar{s}_{i 2}\left(x, \bar{y}(x)^{(r)}, h\right)=\bar{y}_{i}(x)^{(r)}+\frac{h}{16}\left(3 \bar{V}_{i 2}(r)+\bar{V}_{i 2}(r)\right) \\
\underline{s}_{i 3}\left(x ; \underline{y}(x)^{(r)}, h\right)=\underline{y}_{i}(x)^{(r)}+\frac{h}{2} \underline{V}_{i 3}(r), \bar{s}_{i 3}\left(x, \bar{y}(x)^{(r)}, h\right)=\bar{y}_{i}(x)^{(r)}+\frac{h}{2} \bar{V}_{i 3}(r) \\
\underline{s}_{i 4}\left(x ; \underline{y}(x)^{(r)}, h\right)=\underline{y}_{i}(x)^{(r)}+\frac{h}{16}\left(-3 \underline{V}_{i 2}(r)+6 \underline{V}_{i 3}(r)+9 \underline{V}_{i 4}(r)\right) \\
\bar{s}_{i 4}\left(x, \bar{y}(x)^{(r)}, h\right)=\bar{y}_{i}(x)^{(r)}+\frac{h}{16}\left(-3 \bar{V}_{i 2}(r)+6 \bar{V}_{i 3}(r)+9 \bar{V}_{i 4}(r)\right) \\
\underline{s}_{i 5}\left(x ; \underline{y}(x)^{(r)}, h\right)=\underline{y}_{i}(x)^{(r)}+\frac{h}{7}\left(\underline{V}_{i 1}(r)+4 \underline{V}_{i 2}(r)+6 \bar{V}_{i 3}(r)-12 \underline{V}_{i 4}(r)+8 \underline{V}_{i 5}(r)\right) \\
\bar{s}_{i 5}\left(x, \bar{y}(x)^{(r)}, h\right)=\bar{y}_{i}(x)^{(r)}+\frac{h}{7}\left(\bar{V}_{i 1}(r)+4 \bar{V}_{i 2}(r)+6 \bar{V}_{i 3}(r)-12 \bar{V}_{i 4}(r)+8 \bar{V}_{i 5}(r)\right)
\end{array}
$$

where $\underline{V}_{i j}\left(x ; \underline{y}(x)^{(r)}, h\right)=\underline{V}_{i j}(r) \quad$ and $\quad \bar{V}_{i j}\left(x ; \bar{y}(x)^{(r)}, h\right)=\bar{V}_{i j}(r) \quad$ for $j=1,2, \ldots, 6$. Now consider the followings

$$
\begin{aligned}
& L_{i}^{*}\left(x ; \underline{y}(x)^{(r)}, h\right)=7 \underline{V}_{i 1}(r)+32 \underline{V}_{i 3}(r)+12 \underline{V}_{i 4}(r)+32 \underline{V}_{i 5}(r)+7 \underline{V}_{i 6}(r) \\
& U^{*}{ }_{i}\left(x ; \bar{y}(x)^{(r)}, h\right)=7 \bar{V}_{i 1}(r)+32 \bar{V}_{i 3}(r)+12 \bar{V}_{i 4}(r)+32 \bar{V}_{i 5}(r)+7 \bar{V}_{i 6}(r)
\end{aligned}
$$

where $x \in\left[x_{0}, K\right]$ and $r \in[0,1]$. If the exact and numerical solution in the i-th $r$-level at $x_{m}$ for $0 \leq m \leq N$ can be denoted by $\tilde{Y}_{i}(x)^{(r)}=\left[\underline{Y}_{i}(x)^{(r)}, \bar{Y}_{i}(x)^{(r)}\right], \tilde{y}_{i}(x)^{(r)}=\left[\underline{y}_{i}(x)^{(r)}, \bar{y}_{i}(x)^{(r)}\right]$ respectively. According to section (2) the $i$-th coordinate $r$-level set of RK56 is:

$$
\begin{aligned}
& \underline{y}_{i}^{[m+1]}(x)^{(r)}=\underline{y}_{i}^{[m]}(x)^{(r)}+\frac{1}{90} L_{i}^{*}\left(x_{m}, \tilde{y}^{[m]}(x)^{(r)}, h\right), \underline{y}_{i}{ }^{[0]}(r)=\underline{Y}_{i}^{[0]}(r) \\
& \bar{y}_{i}{ }^{[m+1]}(x)^{(r)}=\bar{y}_{i}^{[m]}(x)^{(r)}+\frac{1}{90} U^{*}{ }_{i}\left(x_{m}, \tilde{y}^{[m]}(x)^{(r)}, h\right), \bar{y}_{i}{ }^{[0]}(r)=\bar{Y}_{i}{ }^{[0]}(r)
\end{aligned}
$$

where

$$
\begin{aligned}
& \tilde{y}^{[m]}(x)^{(r)}=\left[\underline{y}^{[m]}(x), \bar{y}^{[m]}(x)\right]_{r}, \underline{y}^{[m]}(r)=\left[\underline{y}_{1}{ }^{[m]}(r), \underline{y}_{2}{ }^{[m]}(r)\right]^{t} \\
& \text { and } \bar{y}^{[m]}(r)=\left[\bar{y}_{1}{ }^{[m]}(r), \bar{y}_{2}{ }^{[m]}(r)\right]^{t}
\end{aligned}
$$

Now we let

$$
L^{*}\left(x_{m}, \tilde{y}^{[m]}(x)^{(r)}, h\right)=\frac{1}{90}\left(L^{*}\left(x_{m}, \tilde{y}^{[m]}(x)^{(r)}, h\right), L_{2}^{*}\left(x_{m}, \tilde{y}^{[m]}(x)^{(r)}, h\right)\right)^{t}
$$




$$
U^{*}\left(x_{m}, \tilde{y}^{[m]}(x)^{(r)}, h\right)=\frac{1}{90}\left(U^{*}{ }_{1}\left(x_{m}, \tilde{y}^{[m]}(x)^{(r)}, h\right), U^{*}{ }_{2}\left(x_{m}, \tilde{y}^{[m]}(x)^{(r)}, h\right)\right)^{t}
$$

The RK5 numerical solution for all $r$-level sets of (17-18) is as follows

$$
\begin{aligned}
& \underline{y}^{[m+1]}(x)^{(r)}=\underline{y}^{[m+1]}(x)^{(r)}+h L^{*}\left(x_{m}, \tilde{y}^{[m]}(x)^{(r)}, h\right), \underline{y}^{[0]}(r)=\underline{Y}^{[0]}(r) \\
& \bar{y}^{[m+1]}(x)^{(r)}=\bar{y}^{[m]}(x)^{(r)}+h U^{*}\left(x_{m}, \tilde{y}^{[m]}(x)^{(r)}, h\right), \bar{y}^{[0]}(r)=\bar{y}^{[0]}(r)
\end{aligned}
$$

and

$$
\begin{aligned}
& L^{*}\left(x_{m}, \tilde{y}^{[m]}(x)^{(r)}, h\right)=\frac{1}{90}\left[7 \underline{V}_{i 1}(r)+32 \underline{V}_{i 3}(r)+12 \underline{V}_{i 4}(r)+32 \underline{V}_{i 5}(r)+7 \underline{V}_{i 6}(r)\right. \\
& U^{*}\left(x_{m}, \tilde{y}^{[m]}(x)^{(r)}, h\right)=\frac{1}{90}\left[7 \bar{V}_{i 1}(r)+32 \bar{V}_{i 3}(r)+12 \bar{V}_{i 4}(r)+32 \bar{V}_{i 5}(r)+7 \bar{V}_{i 6}(r)\right.
\end{aligned}
$$

\section{NUMERICAL EXAMPLE}

Consider the second-order fuzzy nonlinear differential equation [25]:

$$
\begin{aligned}
& y^{\prime \prime}(x)=-\left(y^{\prime}(x)\right)^{2}, \quad 0 \leq x \leq 0.1 \\
& y(0)=(r, 2-r), y^{\prime}(0)=(1+r, 3-r), \forall r \in[0,1]
\end{aligned}
$$

From [25] the exact solution of (25) is:

$$
\left\{\begin{array}{l}
\underline{Y}(x)^{(r)}=\ln \left[\left(e^{r}+e^{r} r\right) x+e^{r}\right] . \\
\bar{Y}(x)^{(r)}=\ln \left[\left(3 e^{2-r}-e^{2-r} r\right) x+e^{2-r}\right] .
\end{array}\right.
$$

Figure 2 is displayed the three-dimensional exact solution (26) corresponding with (25):
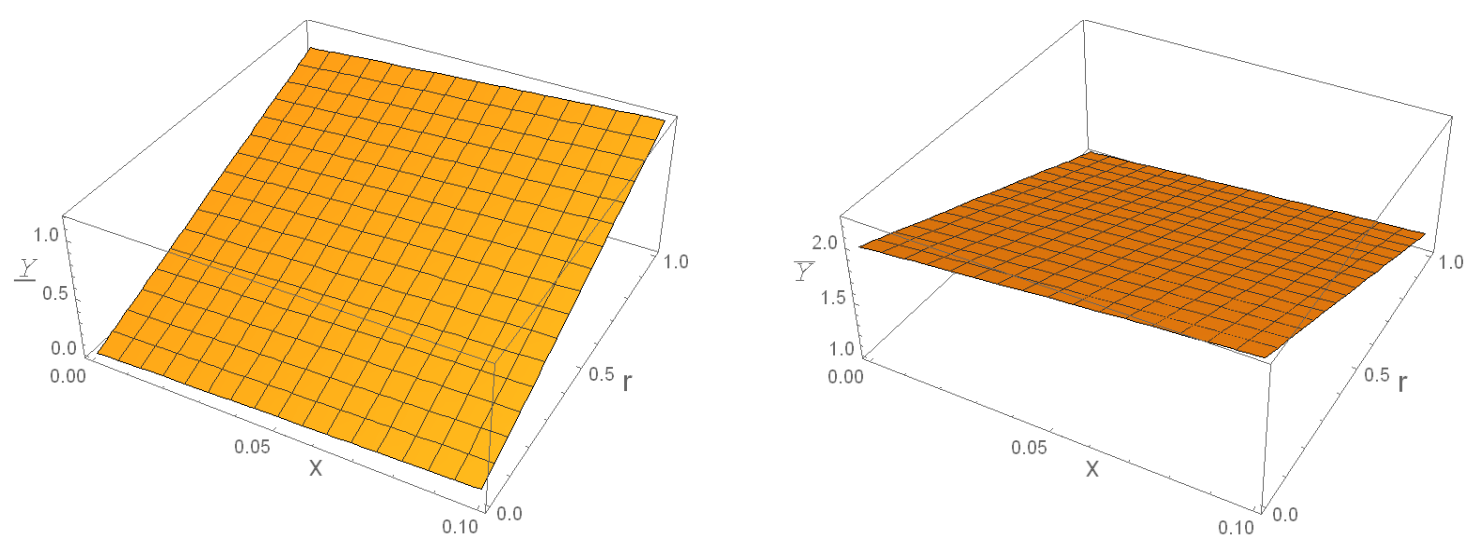

Figure 2. $\underline{Y}(x)^{(r)}$ and $\bar{Y}(x)^{(r)}$ Exact solutions of (25) for all $r \in[0,1]$ and $x \in[0,0.1]$.

From section 3, (25) can be written into first order system as follows

$$
\left\{\begin{array}{c}
\tilde{y}_{1}^{\prime}(x)^{(r)}=\tilde{y}_{2}(x)^{(r)} \\
\tilde{y}_{1}\left(x_{0}\right)^{(r)}=[r, 2-r] \\
\tilde{y}_{2}^{\prime}(x)^{(r)}=-\left[\tilde{y}_{2}(x)^{(r)}\right]^{2} \\
\tilde{y}_{2}\left(x_{0}\right)^{(r)}=[1+r, 3-r]
\end{array}\right.
$$

According to section 4, we apply RK5 to system (26) with selected $h=0.1$ which is enough to obtain convergence results as follows. According to RK56 in section 4, we have: 


$$
\begin{aligned}
& P_{1}\left(x, \tilde{y}_{1}^{\prime}(x)^{(r)}, \tilde{y}_{2}(x)^{(r)}\right)=P_{1}\left(x, \tilde{y}_{1}, \tilde{y}_{2}\right)=\tilde{y}_{2}(x)^{(r)} \\
& P_{2}\left(x, \tilde{y}_{1}^{\prime}(x)^{(r)}, \tilde{y}_{2}(x)^{(r)}\right)=P_{2}\left(x, \tilde{y}_{1}, \tilde{y}_{2}\right)=\left[\tilde{y}_{2}(x)^{(r)}\right]^{2} \\
& \tilde{V}_{1}(r)=h P_{1}\left(x_{i}, \tilde{y}_{1}, \tilde{y}_{2}\right) \\
& \tilde{A}_{1}(r)=h P_{2}\left(x_{i}, \tilde{y}_{1}, \tilde{y}_{2}\right) \\
& \tilde{V}_{2}(r)=h P_{1}\left(x_{i}+\frac{h}{2}, \tilde{y}_{1}+\frac{\tilde{V}_{1}(r)}{2}, \tilde{y}_{2}+\frac{\tilde{A}_{1}(r)}{2}\right) \\
& \tilde{A}_{2}(r)=h P_{2}\left(x_{i}+\frac{h}{2}, \tilde{y}_{1}+\frac{\tilde{V}_{1}(r)}{2}, \tilde{y}_{2}+\frac{\tilde{A}_{1}(r)}{2}\right) \\
& \tilde{V}_{3}(r)=h P_{1}\left(x_{i}+\frac{h}{4}, \tilde{y}_{1}+\frac{3 \tilde{V}_{1}(r)+\tilde{V}_{2}(r)}{2}, \tilde{y}_{2}+\frac{3 \tilde{A}_{1}(r)+\tilde{A}_{2}(r)}{2}\right) \\
& \tilde{A}_{3}(r)=h P_{2}\left(x_{i}+\frac{h}{4}, \tilde{y}_{1}+\frac{3 \tilde{V}_{1}(r)+\tilde{V}_{2}(r)}{2}, \tilde{y}_{2}+\frac{3 \tilde{A}_{1}(r)+\tilde{A}_{2}(r)}{2}\right) \\
& \tilde{V}_{4}(r)=h P_{1}\left(x_{i}+\frac{h}{2}, \tilde{y}_{1}+\frac{\tilde{V}_{3}(r)}{2}, \tilde{y}_{2}+\frac{\tilde{A}_{3}(r)}{2}\right) \\
& \tilde{A}_{4}(r)=h P_{2}\left(x_{i}+\frac{h}{2}, \tilde{y}_{1}+\frac{\tilde{V}_{3}(r)}{2}, \tilde{y}_{2}+\frac{\tilde{A}_{3}(r)}{2}\right) \\
& \left.\quad+\frac{\tilde{A}_{1}(r)+4 \tilde{A}_{2}(r)+6 \tilde{A}_{3}(r)-12 \tilde{A}_{4}(r)+9 \tilde{A}_{5}(r)}{7}\right) \\
& \tilde{V}_{5}(r)=h P_{1}\left(x_{i}+\frac{3 h}{4}, \tilde{y}_{1}+\frac{-3 \tilde{V}_{1}(r)+6 \tilde{V}_{3}(r)+9 \tilde{V}_{4}(r)}{16}, \tilde{y}_{2}+\frac{-3 \tilde{A}_{1}(r)+6 \tilde{A}_{3}(r)+9 \tilde{A}_{4}(r)}{16}\right) \\
& \tilde{D}_{5}(r)=h P_{2}\left(x_{i}+\frac{3 h}{4}, \tilde{y}_{1}+\frac{-3 \tilde{V}_{1}(r)+6 \tilde{V}_{3}(r)+9 \tilde{V}_{4}(r)}{16}, \tilde{y}_{2}+\frac{-3 \tilde{A}_{1}(r)+6 \tilde{A}_{3}(r)+9 \tilde{A}_{4}(r)}{16}\right) \\
& \tilde{V}_{6}(r)=h P_{1}\left(x_{i}+h, \tilde{y}_{1}+\frac{\tilde{V}_{1}(r)+4 \tilde{V}_{2}(r)+6 \tilde{V}_{3}(r)-12 \tilde{V}_{4}(r)+9 \tilde{V}_{5}(r)}{7}, \tilde{y}_{2}\right. \\
& \widetilde{D}_{6}(r)=h P_{2}\left(x+6 \tilde{A}_{3}(r)-12 \tilde{A}_{4}(r)+9 \tilde{A}_{5}(r)\right. \\
& 7
\end{aligned}
$$

then

$$
\begin{aligned}
& \tilde{y}_{1}\left(x_{i+1}\right)^{(r)}=\tilde{y}_{1}\left(x_{i}\right)^{(r)}+\frac{7 \widetilde{V}_{1}(r)+32 \widetilde{V}_{3}(r)+12 \widetilde{V}_{4}(r)+32 \widetilde{V}_{5}(r)+7 \widetilde{V}_{6}(r)}{90} \\
& \tilde{y}_{2}\left(x_{i+1}\right)^{(r)}=\tilde{y}_{2}\left(x_{i}\right)^{(r)}+\frac{7 \tilde{A}_{1}(r)+32 \tilde{A}_{3}(r)+12 \tilde{A}_{4}(r)+32 \tilde{A}_{5}(r)+7 \tilde{A}_{6}(r)}{90}
\end{aligned}
$$

where

$$
\begin{aligned}
& \tilde{y}_{1}\left(t_{i} ; r\right)=\left[\underline{y}_{1}\left(x_{i} ; r\right), \bar{y}_{1}\left(x_{i} ; r\right)\right], \\
& U_{1}\left(x_{i}, \tilde{y}_{1}, \tilde{y}_{2}\right)=\left[U_{1}\left(t_{i}, y_{1}, \underline{y}_{2}\right), U_{1}\left(x_{i}, \bar{y}_{1}, \bar{y}_{2}\right)\right], U_{2}\left(x_{i}, \tilde{y}_{1}, \tilde{y}_{2}\right)=\left[U_{2}\left(x_{i}, \underline{y}_{1}, \underline{y}_{2}\right), U_{2}\left(x_{i}, \bar{x}_{1}, \bar{x}_{2}\right)\right], \\
& \widetilde{K}_{j}(r)=\left[\underline{K}_{j}(r), \bar{K}_{j}(r)\right], \widetilde{D}_{j}(r)=\left[\underline{D}_{j}(r), \bar{D}_{j}(r)\right] \text { and } x_{i+1}=x_{i}+h \text { for } j=1, \ldots, 6, i=1, \ldots, n \\
& \text { and } r \in[0,1] .
\end{aligned}
$$

Define the absolute error of the numerical solution of (25) for all $r \in[0,1]$ as follows

$$
[\underline{E}]_{r}=\left|\underline{Y}(x)^{(r)}-\underline{y}(x)^{(r)}\right|,[\bar{E}]_{r}=\left|\bar{Y}(x)^{(r)}-\bar{y}(x)^{(r)}\right|
$$

From (27-28) we numerical solution of (25) as displayed in Table 1 and Figures 3 and 4:

From Table 1, we can note that the high accuracy of the suggested method in ten step size only and the results or the lower and upper bounds of (25) flow the fuzzy solution as in definition 2.1. Additionally, Figure 3 shows that the RK56 solution over the all (25) given interval and for all the fuzzy level sets from 0 to 1 flow the fuzzy solution as in definition 2.1. Overall, as displayed in Table 1 and Finger 3 we conclude that RK56 numerical solution with 10 iterations solution at $x=0.1$ and for all $0 \leq r \leq 1$ satisfy the fuzzy 
differential equations solution in the form of triangular fuzzy numbers. Figure 4 displayed the compression of the RK56 and exact solutions of (25).

Table 1. RK56 solution and accuracy of (25) at $x=0.1$ with step size $h=0.1$

\begin{tabular}{|c|c|c|c|c|}
\hline$r$ & $\operatorname{RK} 56 y(0.1)^{(r)}$ & $\underline{E} \mathrm{RK} 56$ & $\operatorname{RK56\overline {y}}(0.1)^{(r)}$ & RK56 $\bar{E}$ \\
\hline 0 & 0.095310179804423 & $9.84490267086357 \times 10^{-14}$ & 2.26236426450975 & $4.22675228151092 \times 10^{-11}$ \\
\hline 0.25 & 0.367783035656732 & $3.49276163547074 \times 10^{-13}$ & 1.992946178637013 & $2.66238142643260 \times 10^{-11}$ \\
\hline 0.5 & 0.639761942376130 & $9.71445146547012 \times 10^{-13}$ & 1.723143551330192 & $1.59825486178988 \times 10^{-11}$ \\
\hline 1 & 1.182321556798717 & $4.76285677564192 \times 10^{-12}$ & 1.182321556798717 & $4.76285677564192 \times 10^{-12}$ \\
\hline
\end{tabular}

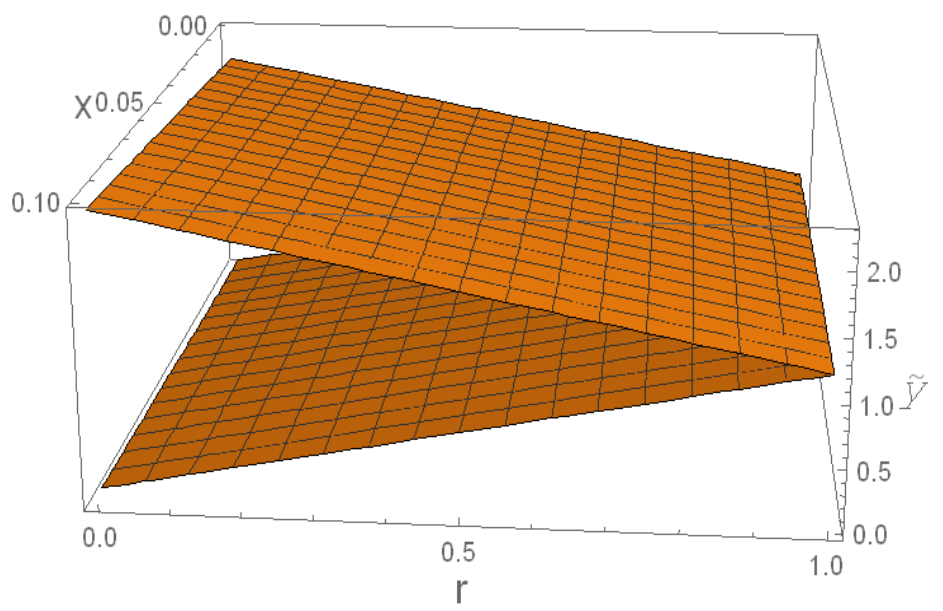

Figure 3. RK 56 numerical solutions of (25) for all $r \in[0,1]$ and $x \in[0,0.1]$

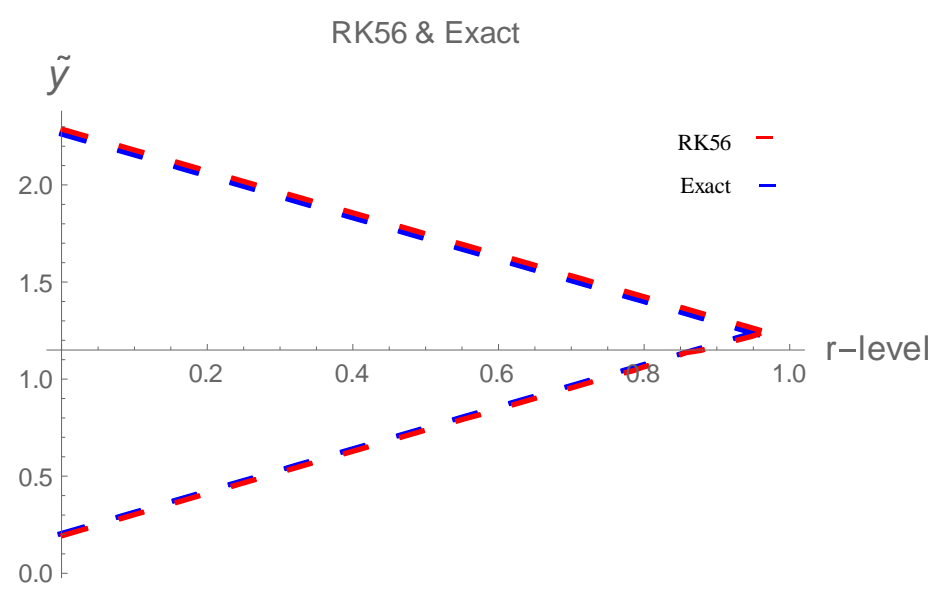

Figure 4. RK 56 and exact solutions of (25) for all $r \in[0,1]$ and $x=0.1$

\section{CONCLUSION}

In this paper a computational approach has been presented and developed for solving fuzzy ordinary differential equations. An approach is based on RK6 for solving nonlinear second order FIVP. A full fuzzy analysis is presented to analyze RK56 to be suitable for solving the proposed problem. Numerical illustration of non-linear second-order FIVP under fuzzy initial conditions demonstrate that the RKM56 is a capable and accurate process with few steps. The findings also by RK56 satisfy the characteristics of fuzzy numbers in triangular shape. 


\section{ACKNOWLEDGEMENTS}

The authors of this research are extremely grateful that they have given us the Fundamental Research Grants Scheme (FRGS) S/O No. 14188 provided by the Higher Education Ministry of Malaysia.

\section{REFERENCES}

[1] The Nemati, K., and Matinfar, M., "An Implicit Method For Fuzzy Parabolic Partial Differential Equations," Journal of Nonlinear Sciences and Applications, vol. 1, no. 2, pp. 61-71, 2008

[2] Kaleva, O., "Fuzzy differential equation," Fuzzy Sets Systems, vol. 24, pp. 301-317, 1987.

[3] Jameel, A. F., Anakira N. R., Alomari, A. K., Hashim, I and Shaher Momani, "A New Approximation Method for Solving Fuzzy Heat Equations," Journal of Computational and Theoretical Nanoscience, vol. 13, no. 11, pp. 7825-7832, 2016.

[4] Anakira N. R., et al., "Direct solution of uncertain bratu initial value problem," International Journal of Electrical and Computer Engineering (IJECE), vol. 9, no. 6, pp. 5075-5083, 2019.

[5] Omer, A., and Omer, O., "A Pray and predator model with fuzzy initial values," Hacettepe Journal of Mathematics and Statistics, vol. 41, no. 3, pp. 387-395, 2013.

[6] Anakira, N. R., et al., "Multistage optimal homotopy asymptotic method for solving initial-value problems," Journal of Nonlinear Science and Applications, vol. 9, no. 4, pp. 1826-1843, 2016.

[7] Smita, T., and Chakraverty, S., "Numerical solution of fuzzy arbitrary order predator-prey equations," Applications And Applied Mathematics, vol. 8, no. 1, pp. 647-673, 2013.

[8] El Naschie, M. S., "From Experimental Quantum Optics to Quantum Gravity Via a Fuzzy Kahler Manifold," Chaos, Solitons \& Fractals, vol. 25, pp. 969-977, 2005.

[9] Abbod, M. F.,Von Keyserlingk, D. G., and Mahfouf, M., "Survey of utilization of fuzzy technology in medicine and healthcare," Fuzzy Sets Systems, vol. 120, pp. 331-3491, 2001.

[10] Julyan, H. E., and Oreste, P., "The dynamics of Runge-Kutta methods," International Journal of Bifurcation and Chaos, vol. 2, pp. 27-449, 1992.

[11] Akbarzadeh, Z., and Mohseni, M., "Solving fuzzy differential equations by Runge-Kutta method," Journal of Mathematics and Computer Science, vol. 2, no. 2, pp. 208-221, 2011.

[12] Abbasbandy, S., Allahvinloo, T., and Darabi, P., "Numerical solution of Nth-order fuzzy differential equations by Runge-Kutta method," Mathematical and Computational Applications, vol. 16, vol. 4, pp. 935-946, 2011.

[13] Parandin, N., "Numerical solution of fuzzy differential equations of 2nd order by Runge-Kutta method," Journal Of Mathematical Extension, vol.7, no. 3, pp. 47-62, 2013.

[14] Jayakumar, T., Kanagarajan, K., and Indrakumar, S., "Numerical solution of Nth-order fuzzy differential equation by Runge-Kutta method of order five," International Journal of Mathematical Analysis, vol. 6, no. 58, pp. 2885-2896, 2012.

[15] Mansour, S. S., and Ahmady, N., "A numerical method for solving Nth-order fuzzy differential equation by using Characterization theorem," Communications in Numerical Analysis, vol. 2012, pp. 1-12, 2012.

[16] Seikkala, S., "On the fuzzy initial value problem," Fuzzy Sets Systems, vol. 24, no, 3, pp. 31-330, 1987.

[17] Fard, O. S., "An iterative scheme for the solution of generalized system of linear fuzzy differential equations," World Applied Sciences Journal, vol. 7, pp. 1597-11604, 2009.

[18] Zadeh, L.A., "Toward a generalized theory of uncertainty," Inform Sciences, vo. 172, no. 2, pp. 1-40, 2005

[19] Faranak, R. Fudziah, I., Ali, A., and Soheil, S., "Numerical solution of second-order fuzzy differential equation using improved Runge-Kutta Nystrom method," Mathematical Problems in Engineering, vol. 2013, pp. 1-10, 2013.

[20] Dubois, D., and Prade, H., "Towards fuzzy differential calculus, Part 3: Differentiation," Fuzzy Sets Systems, vol. 8, pp. 225-233, 1982.

[21] Emre, S., "Comparison of Runge-Kutta methods of order 4 and 5 on Lorenz equation," Journal of Arts and Sciences, vol. 11, pp. 61-69, 2005.

[22] Fae'q, A., and Radwan, A., "Solution of Initial Value problem using fifth order Runge-Kutta method using Excel spreadsheet," Journal of Applied Sciences, vol. 2, no. 1, pp. 44-47, 2002.

[23] Nikolaos, S. C., "An algorithm using Runge-Kutta methods of orders 4 and 5 for systems of ODEs'," International Journal of Numerical Methods and Applications, vol. 2, pp. 47-57, 2009.

[24] Ali, F. J., Ahmad, I. I., and Amir, S., "Numerical solution of fuzzy IVP with trapezoidal and triangular fuzzy numbers by using fifth order Runge-Kutta method," World Applied Sciences Journal, vol. 17, no. 12, pp. 1667-1674, 2012.

[25] Ali F. J, Ghoreishi M., Ahmad Izani Md. Ismai, "Approximate Solution of High Order Fuzzy Initial Value Problems," Journal of Uncertain Systems, vol. 8, no. 2, pp. 149-160, 2014. 